\title{
Cluster Formation Triggered by Filament Collisions in Serpens South
}

\author{
Fumitaka Nakamura ${ }^{1,2,3,4}$, Koji Sugitani ${ }^{5}$, Tomohiro Tanaka ${ }^{6}$, Hiroyuki Nishitani ${ }^{2}$, Kazuhito \\ Dobashi $^{7}$, Tomomi Shimoikura ${ }^{7}$, Yoshito Shimajiri ${ }^{8}$, Ryohei Kawabe ${ }^{1}$ Yoshinori Yonekura $^{9}$, Izumi \\ Mizuno $^{2,11}$, Kimihiko Kimura ${ }^{6}$, Kazuki Tokuda ${ }^{6}$, Minato Kozu ${ }^{6}$, Nozomi Okada ${ }^{6}$, Yutaka \\ Hasegawa $^{6}$, Hideo Ogawa ${ }^{6}$, Seiji Kameno ${ }^{12}$, Hiroko Shinnaga ${ }^{1}$, Munetake Momose ${ }^{13}$, Taku \\ Nakajima ${ }^{14}$, Toshikazu Onishi ${ }^{6}$, Hiroyuki Maezawa ${ }^{6}$, Tomoya Hirota ${ }^{1}$, Shuro Takano ${ }^{2,3}$, Daisuke \\ Iono $^{1}$, Nario Kuno ${ }^{2,3,15}$, Satoshi Yamamoto ${ }^{16}$
}

\begin{abstract}
The Serpens South infrared dark cloud consists of several filamentary ridges, some of which fragment into dense clumps. On the basis of CCS $\left(J_{N}=4_{3}-3_{2}\right), \mathrm{HC}_{3} \mathrm{~N}$ $(J=5-4), \mathrm{N}_{2} \mathrm{H}^{+}(J=1-0)$, and $\mathrm{SiO}(J=2-1, v=0)$ observations, we investigated the kinematics and chemical evolution of these filamentary ridges. We find that CCS is extremely abundant along the main filament in the protocluster clump. We emphasize that Serpens South is the first cluster-forming region where extremely-strong CCS
\end{abstract}

\footnotetext{
${ }^{1}$ National Astronomical Observatory, Mitaka, Tokyo 181-8588, Japan; fumitaka.nakamura@nao.ac.jp

${ }^{2}$ Nobeyama Radio Observatory, Minamimaki, Minamisaku, Nagano 384-1305, Japan

${ }^{3}$ The Graduate University for Advanced Studies (SOKENDAI), 2-21-1 Osawa, Mitaka, Tokyo 181-0015, Japan

${ }^{4}$ Kavli Institute for Theoretical Physics, University of California, Santa Barbara, CA 93106-4030

${ }^{5}$ Graduate School of Natural Sciences, Nagoya City University, Mizuho-ku, Nagoya 467-8501, Japan

${ }^{6}$ Department of Physical Science, Osaka Prefecture University, Gakuen 1-1, Sakai, Osaka 599-8531, Japan

${ }^{7}$ Department of Astronomy and Earth Sciences, Tokyo Gakugei University, Koganei, Tokyo 184-8501, Japan

${ }^{8}$ Laboratoire AIM, CEA/DSM-CNRS-Université Paris Diderot, IRFU/Service d'Astrophysique, CEA Saclay, F91191 Gif-sur-Yvette, France

${ }^{9}$ Center for Astronomy, Ibaraki University, 2-1-1 Bunkyo, Mito, Ibaraki 310-8512, Japan

${ }^{10}$ Institute of Astrophysics and Planetary Sciences, Ibaraki University, Bunkyo 2-1-1, Mito 310-8512, Japan

${ }^{11}$ Department of Physics, Faculty of Science, Kagoshima University, 1-21-35 Korimoto, Kagoshima, Kagoshima 890-0065, Japan

${ }^{12}$ Joint ALMA Observatory, Alonso de Crdova 3107 Vitacura, Santiago, Chile

${ }^{13}$ Institute of Astrophysics and Planetary Sciences, Ibaraki University, Bunkyo 2-1-1, Mito 310-8512, Japan

${ }^{14}$ Solar-Terrestrial Environment Laboratory, Nagoya University, Furo-cho, Chikusa-ku, Nagoya, Aichi 464-8601, Japan

${ }^{15}$ Department of Physics, Graduate School of Pure and Applied Sciences, The University of Tsukuba, 1-1-1 Tennodai, Tsukuba Ibaraki 305-8577, Japan

${ }^{16}$ Department of Physics, Graduate School of Science, The University of Tokyo, Tokyo 113-0033, Japan
} 
emission is detected. The CCS-to- $\mathrm{N}_{2} \mathrm{H}^{+}$abundance ratio is estimated to be about 0.5 toward the protocluster clump, whereas it is about 3 in the other parts of the main filament. We identify six dense ridges with different $V_{\mathrm{LSR}}$. These ridges appear to converge toward the protocluster clump, suggesting that the collisions of these ridges may have triggered cluster formation. The collisions presumably happened within a few $\times 10^{5} \mathrm{yr}$ because CCS is abundant only in such a short time. The short lifetime agrees with the fact that the number fraction of Class I objects, whose typical lifetime is $0.4 \times 10^{5} \mathrm{yr}$, is extremely high as about 70 percent in the protocluster clump. In the northern part, two ridges appear to have partially collided, forming a V-shape clump. In addition, we detected strong bipolar $\mathrm{SiO}$ emission that is due to the molecular outflow blowing out of the protostellar clump, as well as extended weak $\mathrm{SiO}$ emission that may originate from the filament collisions.

Subject headings: ISM: structure — ISM: clouds — ISM: kinematics and dynamics — stars: formation

\section{Introduction}

Over the last few decades, considerable progress has been made in the understanding of star cluster formation (e.g., Lada \& Lada 2003). For example, radio and infrared observations have revealed that star clusters form in parsec-scale dense molecular clumps with masses of order of $10^{2}-10^{3} M_{\odot}$ (Lada \& Lada 2003; Ridge et al. 2003; Battersby et al. 2010). In the clusterforming clumps, stellar feedback plays a crucial role in the structure formation of the clumps (Sandell \& Knee 2001). The clumps tend to keep quasi-virial equilibrium states at least for several free-fall times, by sustaining supersonic turbulence supplied by stellar feedback (e.g., Tan et al. 2006; Li \& Nakamura 2006; Matzner 2007; Nakamura \& Li 2014).

In contrast, the initial conditions of cluster formation remain to be elucidated because of the lack of the observational characterization. This is because once active star formation is initiated, stellar feedback such as protostellar outflows and radiation rapidly shapes their surroundings, making it difficult to track back the physical conditions of the clumps prior to active cluster formation, i.e., pre-protocluster clumps (or, precluster clumps, in short). According to recent numerical simulations, the initial conditions such as density profile, magnetic field distribution, and turbulent Mach number determine some properties of forming clusters (Girichidis et al. 2012; Li et al. 2010) because these factors control the fragmentation process. Therefore, it is important to search for molecular clouds where active cluster formation have just been initiated or is just about to be initiated.

Recently, Tanaka et al. (2013) found that the Serpens South infrared dark cloud (IRDC, $d \sim$ $415 \mathrm{pc}$ ) is the region that contains both extremely-young protocluster and precluster clumps. The Serpens South IRDC is a filamentary molecular cloud that contains several filamentary ridges 
Gutermuth et al. 2008; André et al. 2010; Maury et al. 2011; Kirk et al. 2013; Arzoumanian et al. 2013). The central clump is associated with a very young $(\lesssim 0.5 \mathrm{Myr})$ cluster of low-mass protostars (Gutermuth et al. 2008) and is most massive with about $230 M_{\odot}$ (Tanaka et al. 2013). A northern clump shows no sign of active star formation, although its mass $\left(\sim 200 M_{\odot}\right)$ and size are comparable to those of the central protocluster clump, i.e., this northern clump is the precluster clump. Both protocluster and precluster clumps have virial ratios as small as 0.2 , indicating that the internal turbulent motions cannot support the whole clumps against gravity. In spite of the small virial ratios, the clumps do not exhibit rapid infall motions, and therefore their global contraction is likely to be delayed by the moderately-strong magnetic fields that are perpendicular to the filament axis. From the Chandrasekhar-Fermi method, the field strength is estimated to be a few $\times 10^{2} \mu \mathrm{G}$, close to the critical value above which the cloud can be supported against collapse (Sugitani et al. 2011).

In the present paper, we aim to unveil how cluster formation has been initiated in Serpens South, by investigating the kinematics of filamentary ridges on the basis of CCS and $\mathrm{N}_{2} \mathrm{H}^{+}$observations. CCS and $\mathrm{N}_{2} \mathrm{H}^{+}$are known to be abundant in early and late phases of prestellar evolution, respectively, and therefore are appropriate to inspect the evolutionary stages of young star-forming regions (e.g., Hirota et al. 2009).

\section{Observations and Data}

We carried out CCS $J_{N}=4_{3}-3_{2}(45.379033 \mathrm{GHz})$ and $\mathrm{HC}_{3} \mathrm{~N} J=5-4(45.490316 \mathrm{GHz})$ mapping observations toward Serpens South in 2013 May with the Nobeyama 45-m telescope. We use a new $45 \mathrm{GHz}$ band, dual-polarization receiver, Z45 (Tokuda et al. 2013). The main beam efficiency and Half Power Beam Width (HPBW) were $\eta_{\mathrm{Z} 45} \simeq 0.7$ and $\Theta \simeq 37^{\prime \prime}$, respectively. At the back end, we used the 4 sets of 4096 channel SAM45 spectrometer whose frequency resolution was set to $3.81 \mathrm{kHz}\left(\approx 0.025 \mathrm{~km} \mathrm{~s}^{-1}\right.$ at $\left.45 \mathrm{GHz}\right)$, The intensity calibration was made by observing the cyanopolyyne peak of TMC-1 using the S40 receiver + AOS system.

We also carried out $\mathrm{SiO} J=2-1(86.847010 \mathrm{GHz})$ observations in 2013 May using the $\mathrm{TZ}+$ SAM45 receiver system of the $45-\mathrm{m}$ telescope. The main beam efficiency and HPBW were $\eta_{\mathrm{TZ}} \simeq$ 0.36 and $19^{\prime \prime}$ at $86 \mathrm{GHz}$, respectively (Nakajima et al. 2013).

The typical system temperatures during the observations were $150 \mathrm{~K}$ and $120 \mathrm{~K}$ for Z45 and TZ, respectively. The telescope pointing was checked every 1 hour by observing a SiO maser source, IRC +00363 , and the typical pointing offset was better than $3^{\prime \prime}$ during the whole observing period. By applying a convolution scheme with a spheroidal function, the final maps were obtained by combining x-scan and y-scan OTF data into single maps with grid sizes of $15^{\prime \prime}$ and $7.5^{\prime \prime}$ for the Z45 and TZ observations, respectively. The resultant effective resolutions of the maps were about $49^{\prime \prime}$ and $25^{\prime \prime}$ for Z45 and TZ, respectively. In the following, the intensities are shown in the main beam brightness temperature units, $T_{\mathrm{mb}}=T_{\mathrm{A}}^{*} / \eta_{\mathrm{Z} 45}$ or $T_{\mathrm{A}}^{*} / \eta_{\mathrm{TZ}}$, where $T_{\mathrm{A}}^{*}$ is the antenna temperature corrected for the atmospheric attenuation. The rms noise levels are $0.32 \mathrm{~K}$ and $0.24 \mathrm{~K}$ at the 
velocity resolution of $0.05 \mathrm{~km} \mathrm{~s}^{-1}$ for $\mathrm{Z} 45$ and TZ, respectively.

For the $\mathrm{N}_{2} \mathrm{H}^{+}$data, we used the data presented in Tanaka et al. (2013).

\section{Global Gas Distribution and Kinematics}

In Serpens South, several less-dense filamentary ridges appear to converge toward the protocluster clump (see also Myers 2009). In Figure 1(a), we show the positions of nine representative filamentary ridges that are overlaid on the $\mathrm{H}_{2}$ column density map obtained by Herschel data with $36^{\prime \prime} .3$ effective resolution (see Tanaka et al. 2013). The positions of the ridges are determined by eye from the Herschel map. The identified ridges are named as F1, F2, ․, F9 in Figure 1(a).

F2 and F8 trace the main filamentary cloud. F2 and F3 appear to be connected with each other at their southern tips, creating the V-shape precluster clump. F1 is located between F2 and F3. F2, F3, and F8 are essentially the same as those identified by Kirk et al. (2013) who found the large velocity gradient of $2.2 \pm 0.3 \mathrm{~km} \mathrm{~s}^{-1} \mathrm{pc}^{-1}$ along $\mathrm{F} 8$, where we adopted the distance of 415 pc. F7 is extended toward the eastward direction and can also be recognized in the Spitzer IRAC image (see Figure 1 of Gutermuth et al. 2008).

The CCS, $\mathrm{HC}_{3} \mathrm{~N}$, and $\mathrm{N}_{2} \mathrm{H}^{+}$integrated intensity maps show that their emission traces reasonably well the dense parts of the cloud (Figures. 1(b) though 1(d)). F1, F2, F3, F7, F8, and F9 are detected in $\mathrm{N}_{2} \mathrm{H}^{+}, \mathrm{HC}_{3} \mathrm{~N}$, and/or CCS, and thus contain dense gas with densities of $10^{4}-10^{5}$ $\mathrm{cm}^{-3}$. Other ridges, F4, F5, and $\mathrm{F} 6$, are not clearly detected in $\mathrm{CCS}, \mathrm{HC}_{3} \mathrm{~N}$, and $\mathrm{N}_{2} \mathrm{H}^{+}$. The CCS emission tends to be strong along the main filamentary cloud F2+F8. In particular, the CCS emission is extremely strong along F2, whereas it is weak in the protocluster clump where the $\mathrm{N}_{2} \mathrm{H}^{+}$emission is strongest. The CCS column density is estimated to be about $\sim 1 \times 10^{14} \mathrm{~cm}^{-2}$ toward the precluster clump. Such an extremely high column density of CCS is typical of the Carbon-Chain Producing Regions suggested by Hirota et al. (2009).

The CCS fractional abundances are estimated to be about $1 \times 10^{-9}, 1 \times 10^{-10}$, and $1 \times 10^{-9}$ toward the dense part of F8, protocluster clump, and precluster clump, respectively, where we adopted the excitation temperature of $5 \mathrm{~K}$ and LTE approximation (e.g., Wolkovitch et al. 1997; Lai \& Crutcher 2000). We note that a change in excitation temperature of $1 \mathrm{~K}$ causes a change in column density and abundance of $30 \%$. The CCS fractional abundance in the protocluster clump is of the order of magnitude smaller than the other parts of the main filament. The $[\mathrm{CCS}] /\left[\mathrm{N}_{2} \mathrm{H}^{+}\right]$ abundance ratio are also estimated to be 3.0, 0.4, and 2.5, for F8, protocluster clump, and precluster clump, respectively, where $\mathrm{N}_{2} \mathrm{H}^{+}$abundances are adopted from Tanaka et al. (2013). In other words, the distribution of CCS tends to be anti-correlated with that of $\mathrm{N}_{2} \mathrm{H}^{+}$. According to the chemical evolution (e.g., Suzuki et al. 1992; Marka et al. 2012), CCS is abundant only in the early phase of prestellar evolution $\left(\lesssim\right.$ a few $\left.\times 10^{5} \mathrm{yr}\right)$, whereas $\mathrm{N}_{2} \mathrm{H}^{+}$becomes abundant in the late phase. Thus, CCS and $\mathrm{N}_{2} \mathrm{H}^{+}$can often been used as an age indicator of dense cores (e.g., de Gregorio-Monsalvo et al. 2006; Sakai et al. 2007; Hirota et al. 2009; Devine et al. 2011). The 
CCS-rich F2 and F8 ridges are likely to be chemically and dynamically young. $\mathrm{HC}_{3} \mathrm{~N}$ distribution is roughly similar to that of CCS.

To see the global kinematic motions of the filamentary ridges, we show in Figures 2 (a) and 2 (b) the centroid velocity maps of $\mathrm{N}_{2} \mathrm{H}^{+}$and CCS, respectively, which are overlaid on the Herschel $\mathrm{H}_{2}$ column density contour map. The CCS and $\mathrm{N}_{2} \mathrm{H}^{+}$centroid velocity maps are in good agreement with each other, except at the central clump. The maps show that the main filamentary ridge, F8, is blueshifted from the systemic velocity of the central protocluster clump $\left(V_{\text {sys }} \simeq 7 \mathrm{~km} \mathrm{~s}^{-1}\right)$.

On the other hand, other ridges, F2, F3, F7, and F9, are redshifted from $V_{\text {sys }}$ (Figures. 2 (a) and 2 (b).) The velocity structures in the northern part are somewhat complicated. The typical difference in $V_{\mathrm{LSR}}$ is around $0.5-0.7 \mathrm{~km} \mathrm{~s}^{-1}$ in the northern part. The dynamical time of the filament suggests that the filaments are formed with short spacing of $0.4-0.5$ pc $(\approx$ $\left.0.5 \mathrm{Myr} \times(0.5-0.7) \mathrm{km} \mathrm{s}^{-1} / \cos 45^{\circ}\right)$, where we assume that the collision velocity is equal to the velocity difference and this region has an inclination of $45^{\circ}$. Here we adopt the dynamical time of about 0.5 Myr, which is the lifetime of Serpens South protocluster. This suggests that the filaments do not form by gravitational fragmentation, instead by external events such as turbulent compression, and the densities become as high as $10^{4} \mathrm{~cm}^{-3}$ within a few $\times 10^{5} \mathrm{Myr}$.

In fact, the CCS line profiles shown in Figures 2 (c) through 2 (f) consist of a couple of components with different velocities in several positions around the V-shape clump. The CCS line width of each component is typically $0.6-0.8 \mathrm{~km} \mathrm{~s}^{-1}$, which is about $1.5-2$ times larger than the

typical CCS line widths $\left(0.3-0.6 \mathrm{~km} \mathrm{~s}^{-1}\right)$ of more quiescent clouds such as Taurus (Suzuki et al. 1992).

The main ridge F8 has a significant velocity gradient that can be interpreted as an infall motion toward the protocluster clump (Kirk et al. 2013), although the northern part of the filament does not show similar velocity gradients. On the other hand, CCS is weak along F7 and F9. It is worth noting that Serpens South is a really unique cluster-forming region in terms of the extremely-high fractional abundance of CCS and other carbon-chain molecules such as $\mathrm{HC}_{7} \mathrm{~N}$ (Friesen et al. 2013). The CCS line widths are also wider than those of nearby CCS-rich dark clouds such as Taurus.

\section{A Scenario of Triggered Cluster Formation in Serpens South}

From these characteristics, we propose the following scenario of cluster formation in Serpens South, as in Figure 3 .

In Serpens South, the magnetic field is relatively strong and plays an important role in cloud dynamics (Sugitani et al. 2011). In the parent cloud, multiple filamentary ridges were created due to internal MHD turbulence and/or large-scale flows. These filamentary ridges were preferentially perpendicular to the global magnetic field lines and moved along the magnetic field lines. The filaments also fragmented into several subclumps. Since the filaments were likely to be magneti- 
cally supported (Sugitani et al. 2011), the fragmentation was presumably triggered by ambipolar diffusion accelerated in a turbulent environment (Nakamura \& Li 2008; Tanaka et al. 2013). The collisions tend to make the effective magnetic support reduced and inject additional turbulence in the dense parts. Consequently, cluster formation was initiated in the central clump.

It is difficult to identify which ridges are paired with which because the velocity structure is complicated particularly in the northern part. But, we suggest that F2 and F8 were from the same filament because CCS is abundant along these ridges. This identification agrees with the structure identified by Kirk et al. (2013). Kirk et al. (2013) also suggested that F3 is paired with F9. If this is the case, F1 and F7 might be from the same filament. But, there is a possibility that F1 and F3 may be paired with F9 and F7, respectively.

In any of these cases, at least three filamentary ridges may have collided at the position of the protocluster clump. The collision may have happened within a few $\times 10^{5} \mathrm{yr}$ because most of the protostars in the dense clump are the Class I objects whose typical lifetime is around 0.4 Myr (Evans et al. 2009). This timescale is in good agreement with that inferred from the chemical evolution of CCS. Two subclumps formed along the different ridges F2 and F3 have just collided with each other to form the V-shape clump, which may be on the verge of future cluster formation. Such dynamical event may have caused the CCS line widths to be wider than those in more quiescent dark clouds.

To search for further evidence of the filament collisions, we carried out $\operatorname{SiO}(J=2-1$, $v=0$ ) observations. The $\mathrm{SiO}$ emission is sometimes used as a shock tracer of the cloud-cloud collision (e.g., Jimenez-Serra et al. 2010). Figure 2 (j) shows the SiO integrated intensity map. We detected emission from both the central protocluster and northern precluster clumps. We note that Kirk et al. (2013) reported no detection of $\mathrm{SiO}$ emission on the basis of Mopra 22-m observations. This apparent discrepancy comes from the fact that the sensitivity of our map is much better than that of Kirk et al. (2013). The emission associated with the central clump is likely to be due to a protostellar outflow, because the emission shows a well-defined bipolar feature. In addition, we detected weak emission lines at several positions as presented in Figures 2 (g) through 2 (d). These weak $\mathrm{SiO}$ lines might contain contribution of compressional heating by the ongoing filament collision at around $V_{\mathrm{LSR}} \sim 7 \mathrm{~km} \mathrm{~s}^{-1}$. However, the higher velocity component $\left(V_{\mathrm{LSR}} \gtrsim 10 \mathrm{~km} \mathrm{~s}^{-1}\right)$ near the southern tip of the northern clump presumably originates from the interaction between the northern clump and the redshifted CO lobe identified by Nakamura et al. (2011) as R2, because the $\mathrm{SiO}$ emission overlaps well with the northern part of the redshifted CO lobe whose velocity range is $V_{\mathrm{LSR}} \sim 10-30 \mathrm{~km} \mathrm{~s}^{-1}$. Further justification is needed to confirm the origin of the weak $\mathrm{SiO}$ emission.

Position-velocity diagrams presented in Figures. 4 (a) though 4 (c) show two components with different $V_{\mathrm{LSR}}$ in the northern part. These two appear to converge at the precluster clump where line width becomes wider (Figure. [4 (c)). Such structure is reminiscent of the kinetic structure generated by filament collision (Duarte-Cabral et al. 2011). At the protocluster clump, there also 
appear two components with different $V_{\mathrm{LSR}}: 6.5 \mathrm{~km} \mathrm{~s}^{-1}$ and $7.5 \mathrm{~km} \mathrm{~s}^{-1}$. The latter is weak and vaguely recognized in the position-velocity diagrams. This component might be a remnant of F9 due to filament collision. A large velocity gradient along F8 appears inconsistent with an accretion flow model (Kirk et al. 2013) because the accretion flow model predicts that the velocity gradient near the clump should be largest because the flow is accelerated by self-gravity and at both sides of the clump, infall motions should be observed (Figure 5d of Nakamura et al. (1991)). Thus, we conclude that our collision scenario appears more consistent with kinetic structure of Serpens South than an accretion model.

Recently, the cloud-cloud collision has paid attention as a promising mechanism for triggering cluster formation (e.g., Tasker \& Tan 2009; Higuchi et al. 2010; Shimoikura et al. 2013; Fukui et al. 2014; Jimenez-Serra et al. 2014). Serpens South is the nearest IRDC that contains both protocluster and precluster clumps. Therefore, it is an interesting region to investigate how the cloud-cloud collision triggers individual star formation by using high-angular resolution interferometric observations such as ALMA.

This work is supported in part by a Grant-in-Aid for Scientific Research of Japan (24244017) and the National Science Foundation under Grant (NSF PHY11-25915). We are grateful to the staffs at the Nobeyama Radio Observatory for both operating the 45-m and helping us with the data reduction. NRO is a branch of the National Astronomical Observatory, National Institutes of Natural Sciences, Japan.

\section{REFERENCES}

André, P., Menshchikov, A., Bontemps, S. et al. 2010, A\&A, 518, L102

Arzoumanian, D., André, P., Peretto, N., \& Könyves, V. 2013, A\&A, 553, 119

Battersby, C., Bally, J., Jackson, J. M. et al. 2010, ApJ, 721, 222

Duarte-Cabral, A., Dobbs, C. L., Peretto, N., \& Fuller G. A. 2011, A\&A, 528, 50

de Gregorio-Monsalvo, I., Goḿez, J. F., Suárez, O. et al. 2006, ApJ, 642, 319

Devine, K. E., Chandler, C. J., Brogan, C., et al. 2011, ApJ, 733, 44

Evans, N. J. II, Dunham, M. M., Jorgensen, J. K., et al. 2009, ApJS, 181, 321

Friesen, R. K., Medeiros, L., Schnee, S. et al. 2013, MNRAS, 436, 1513

Fukui, Y., Ohama, A., Hanaoka, N. et al. 2014, ApJ, 780, 36

Gutermuth, R. A., Bourke, T. L., Allen, L. E., et al. ApJ, 2008, 673, L151 
Girichidis, P., Federrath, C., Allison, R. et al. 2012, MNRAS, 420, 3264

Higuchi, A. E., Kurono, Y., Saito, M., Kawabe, R. 2010, ApJ, 719, 1813

Hirota, T., Ohishi, M., \& Yamamoto, S. 2009, ApJ, 699, 585

Jimenez-Serra, I., Caselli, P., Tan, J. C., et al. 2010, MNRAS, 406, 187

Jimenez-Serra, I., Caselli, P., Fontani, F. et al. 2014, MNRAS, in press (arXiv:1401.2347)

Kirk, H., Myers, P. C., Bourke, T. L., et al. 2013, ApJ, 766, 115

Lada, C. J. \& Lada, E. A. 2003, ARA\&A, 31, 57

Lai, S.-P. \& Crutcher, R. M. 2000, ApJ, 128, 271

Larson, R. B. 1981, MNRAS, 194, 809

Li, Z.-Y., \& Nakamura, F. 2006, ApJ, 640, L187

Li, Z.-Y., Wang, P., Abel, T., \& Nakamura, F. 2010, ApJ, 720, L26

Marka, C., Schreyer, K., Launhardt, R., et al. 2012, A\&A, 537, A4

Matzner, C. D. 2007, ApJ, 659, 1394

Maury, A., André, P., Men'shchikov, A., Könyves, V., \& Bontemps, S. 2011, A\&A, 535, 77

McKee, C. F., \& Ostriker, E. C. 2007, ARA\&A, 45, 565

Myers, P. C. 2009, ApJ, 700, 1609

Nakamura, F.AHanawa, T., \& Nakano, T. 1991, ApJ, 444, 770

Nakamura, F. \& Li, Z.-Y. 2008, ApJ, 687, 354

Nakamura, F. \& Li, Z.-Y. 2014, ApJ, in press

Nakamura, F., Sugitani, K., Shimajiri, Y. et al. 2011, ApJ, 737, 56

Nakajima, T., Kimura, K., Nishimura, A. et al. 2013, PASP, 125, 252

Ridge, N. A., Wilson, T. L., Megeath, S. T., et al. 2003, AJ, 126, 286

Sakai, T., Oka, T., Yamamoto, S. 2007, ApJ, 662, 1043

Sandell, G., \& Knee, L. 2001, ApJ, 546, L49

Shimoikura, T., Dobashi, K., Saito, H., et al. 2013, ApJ, 768, 72

Sugitani, K., Nakamura, F., Watanabe, M. et al. 2011, ApJ, 734, 63 
Suzuki, H., Yamamoto, S., Ohishi, M., et al. 1992, ApJ, 392, 551

Tan, J. C., Krumholz, M. R., \& McKee, C. F. 2006, ApJ, 638, 369

Tasker, E. J., \& Tan, J. C. 2009, ApJ, 700, 358

Tanaka, T., Nakamura, F., Awazu, Y. et al. 2013, ApJ, 778, 34

Tokuda, K., Kozu, M., Kimura, K., et al. 2013, Astronomical Society of the Pacific Conference Series, 476, 403

Wolkovitch, D., Langer, W. D., Goldsmith, P. F., \& Heyer, M. 1997, ApJ, 477, 241

This preprint was prepared with the AAS $\mathrm{LAT}_{\mathrm{E}} \mathrm{X}$ macros v5.2. 

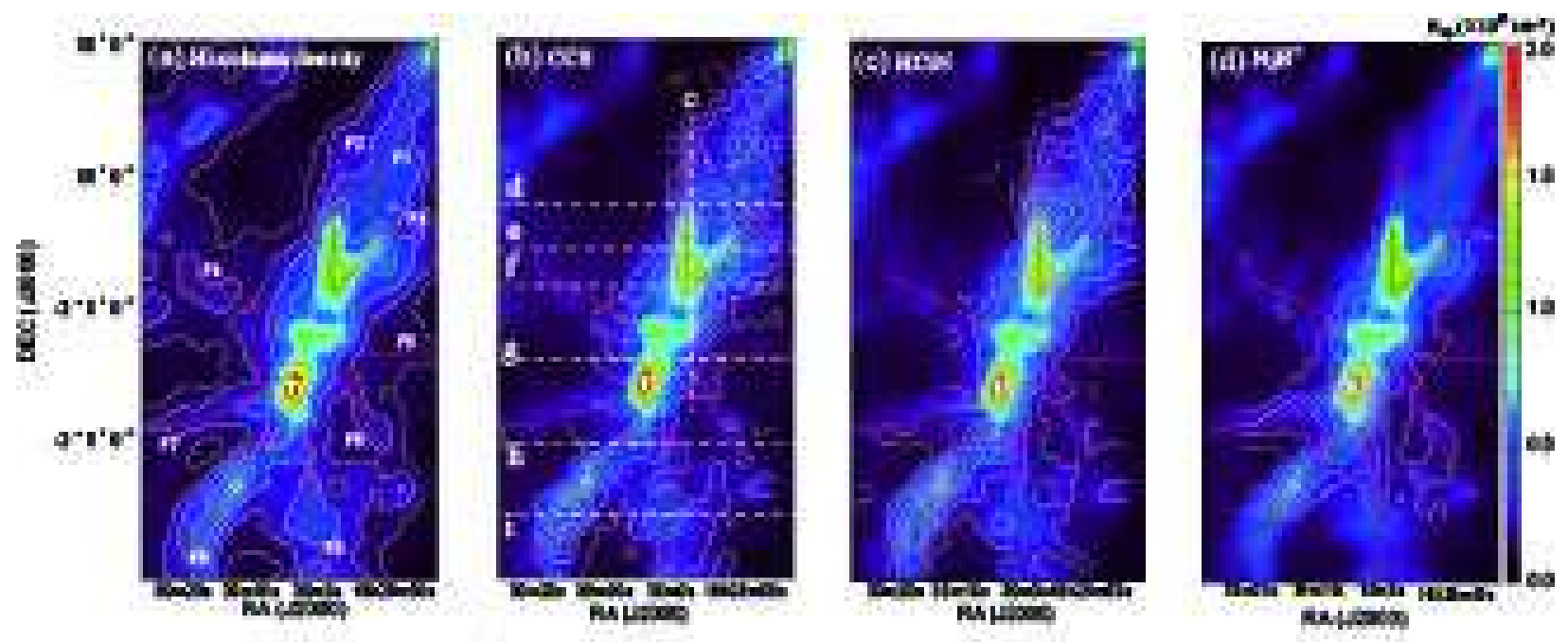

Fig. 1.- (a) The $\mathrm{H}_{2}$ column density map (see Tanaka et al. 2013 for detail.) The positions of nine ridges are indicated with the dashed curves. The contours start at $0.12 \times 10^{23} \mathrm{~cm}^{-2}$ with a logarithmic interval of $\log \left[\Delta N_{\mathrm{H}_{2}} /\left(1.2 \times 10^{23} \mathrm{~cm}^{-2}\right)\right]=0.2$. (b) CCS $\left(J_{N}=4_{3}-3_{2}\right)$ integrated intensity contour map in the velocity range from $V_{\mathrm{LSR}}=5.5$ to $9.0 \mathrm{~km} \mathrm{~s}^{-1}$. The image is overlaid on the $\mathrm{H}_{2}$ column density map. The contour level starts from $0.33 \mathrm{~K} \mathrm{~km} \mathrm{~s}^{-1}$ with an interval of $0.33 \mathrm{~K} \mathrm{~km} \mathrm{~s}^{-1}$, corresponding to the $3 \sigma$ noise level. (c) $\mathrm{HC}_{3} \mathrm{~N}(J=5-4)$ integrated intensity contour map in the velocity range from $V_{\mathrm{LSR}}=5.5$ to $9.0 \mathrm{~km} \mathrm{~s}^{-1}$. The image is overlaid on the $\mathrm{H}_{2}$ column density map. The contour level starts from $0.3 \mathrm{~K} \mathrm{~km} \mathrm{~s}^{-1}$ with an interval of $0.4 \mathrm{~K} \mathrm{~km} \mathrm{~s}^{-1}$. (d) $\mathrm{N}_{2} \mathrm{H}^{+}(J=1-0)$ integrated intensity contour map overlaid on the $\mathrm{H}_{2}$ column density map. The contour level starts from $2 \mathrm{~K} \mathrm{~km} \mathrm{~s}^{-1}$ with an interval of $2 \mathrm{~K} \mathrm{~km} \mathrm{~s}^{-1}$. 

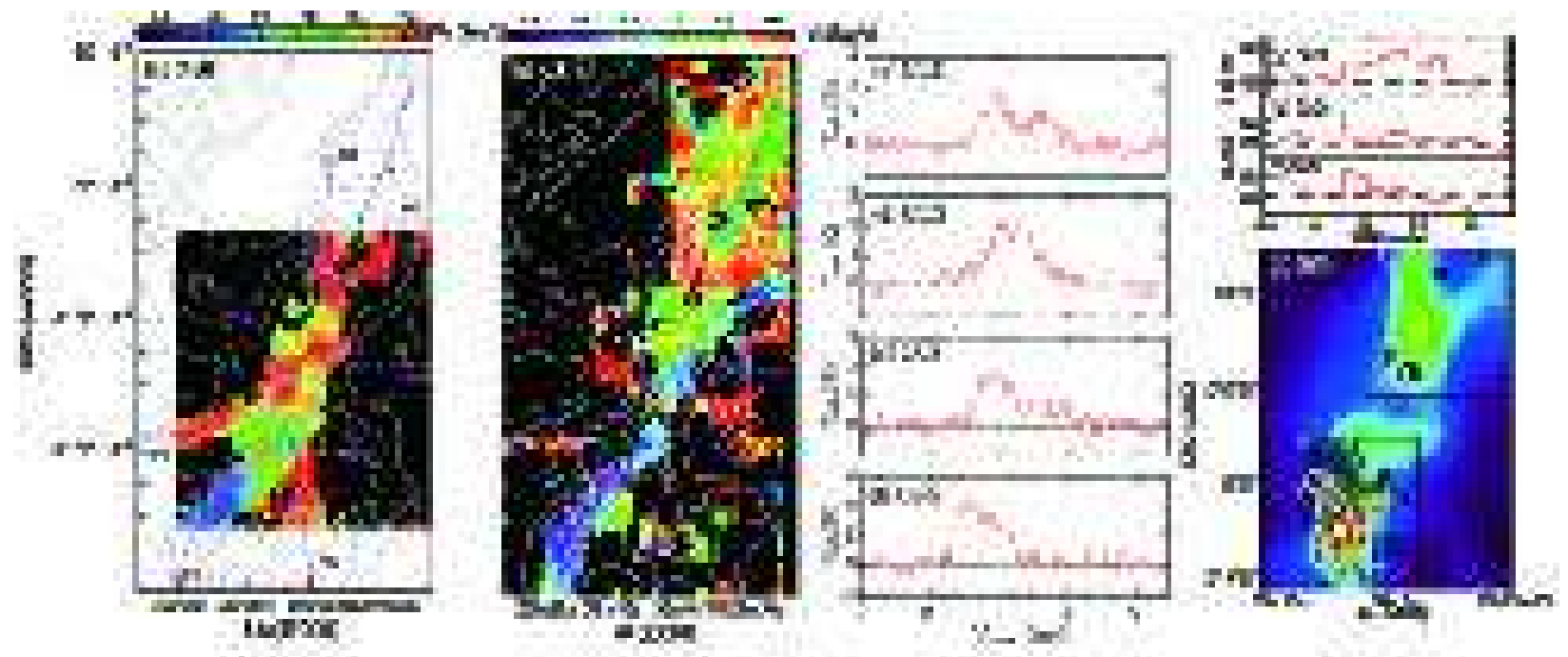

Fig. 2.- (a) Centroid velocity map for $\mathrm{N}_{2} \mathrm{H}^{+}(J=1-0)$ across Serpens South. The $\mathrm{N}_{2} \mathrm{H}^{+}$centroid velocity map was obtained by fitting the seven hyperfine lines with Gaussian profiles (Tanaka et al. 2013). (b) same as panel (a) but for CCS $\left(J_{N}=4_{3}-3_{2}\right)$. For panels (a) and (b), the contours are the same as those in Fig. 1 (a). (c) through (f) CCS line profiles at four positions that are designated in Fig. 2 $\mathrm{b}$ with circles. (g) $\mathrm{SiO}(J=2-1, v=0)$ line profile at the position $\mathrm{g}$ in panel (j). (h) Same as panel (g) but for the position h. (i) Same as panel (g) but for the position i. (j) $\mathrm{SiO}(J=2-1)$ integrated intensity contour map overlaid on the $\mathrm{H}_{2}$ column density map. The black and gray contours show the blueshifted and redshifted components integrated over $-10 \sim 7$ $\mathrm{km} \mathrm{s}^{-1}$ and $8 \sim 20 \mathrm{~km} \mathrm{~s}^{-1}$, respectively. The contour level starts from $1 \mathrm{~K} \mathrm{~km} \mathrm{~s}^{-1}$ with an interval of $1 \mathrm{~K} \mathrm{~km} \mathrm{~s}^{-1}$, corresponding to the $3.5 \sigma$ and $2.5 \sigma$ noise levels for the blueshifted and redshifted components, respectively. The color image is the same as that shown in Figure 1. 


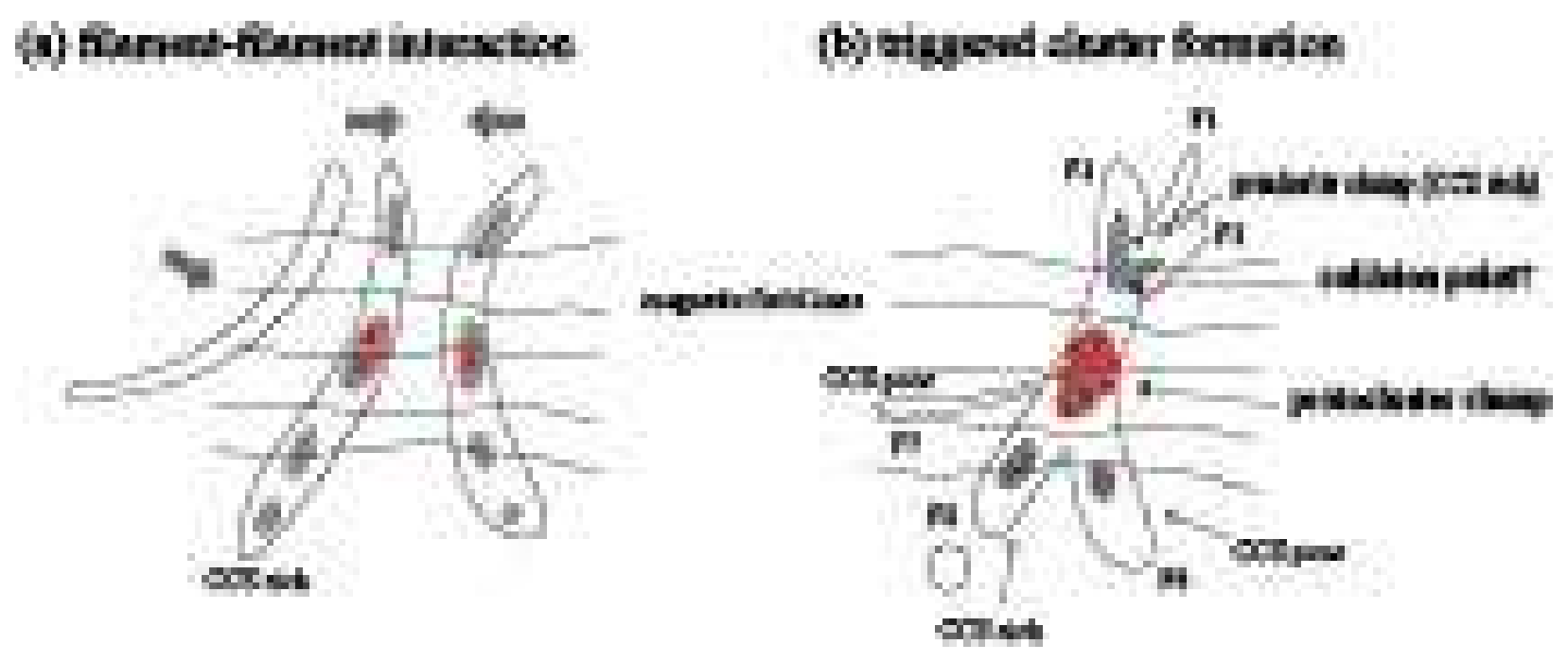

Fig. 3.- Possible scenario of cluster formation triggerd by filament collisions in Serpens South. (a) at least three filamentary flows are on the verge of the collisions along the global magnetic field lines. The filaments fragment into several subclumps due to turbulence-accelerated ambipolar diffusion. Several stars may have formed in the subclumps before the collisions, and these stars are now observed as Class II sources. (b) They collided and crossed at the center where cluster formation was triggered. For the northern clump, the collision has just happened at its southern tip. 


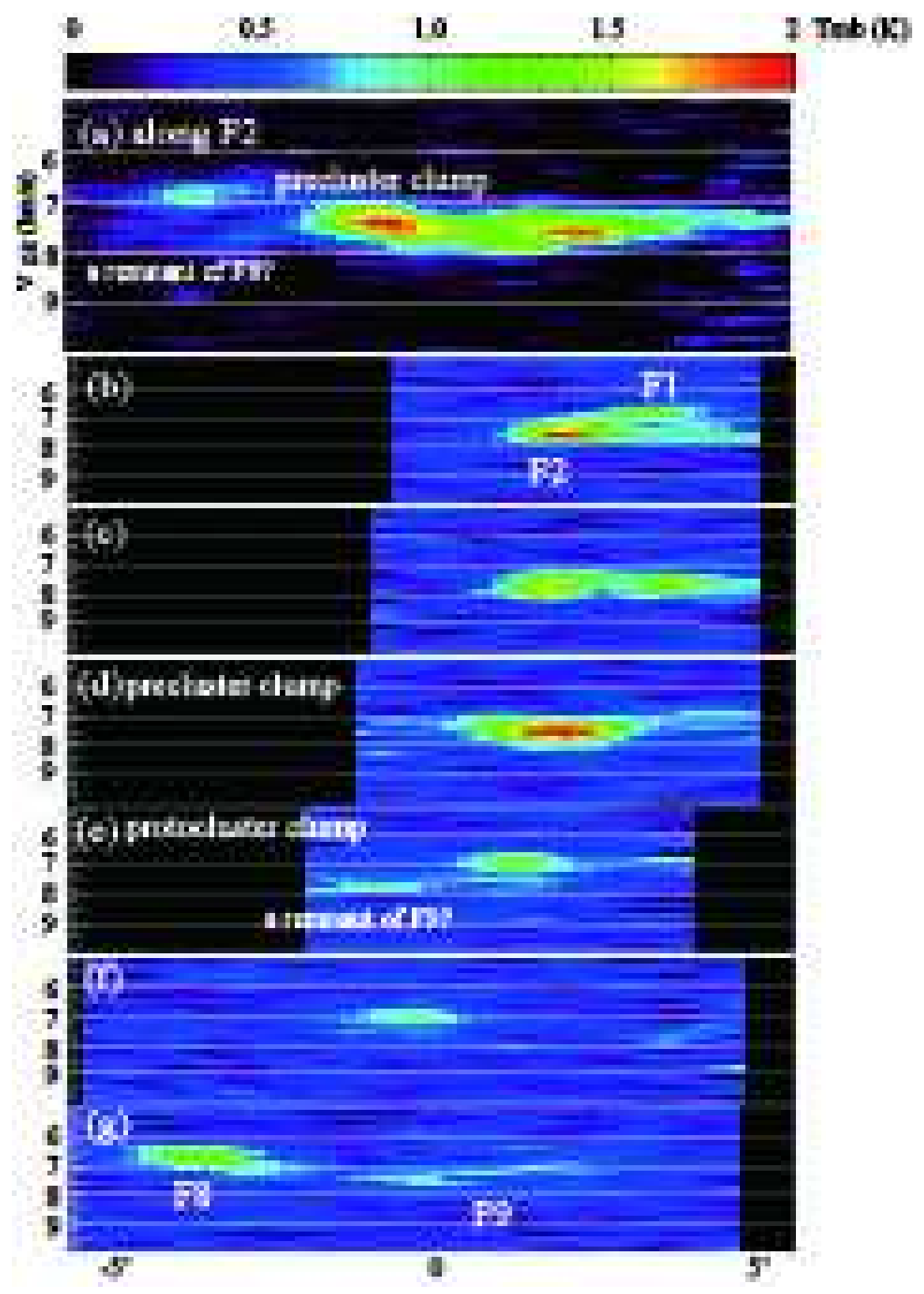

Fig. 4.- Position-velocity diagram of CCS. The positions of these cuts in the map are shown as white dashed lines in 